\title{
Visualization of chromatin folding patterns in chicken erythrocytes by atomic force microscopy (AFM)
}

\author{
QIAN RUO LAN ${ }^{1}$ Zheng Xia LIU, Mei Yun ZHOU, \\ HeN Yue XIE, CHU JIANG, ZHI JIANG YAN \\ Shanghai Institute of Cell Biology, Chinese Academy of \\ Sciences, Shanghai 200031, China \\ LI MIN QIAN, Yi ZHANG, JUN HU \\ Shanghai Institute of Nuclear Research, Chinese Academy \\ of Sciences, Shanghai 201800, China
}

\begin{abstract}
The organization of the higher order structure of chromatin in chicken erythrocytes has been examined with tapping-mode scanning force microscopy under conditions close to their native environment. Reproducible highresolution AFM images of chromatin compaction at several levels can be demonstrated. An extended beads-on-astring (width of $\sim 15-20 \mathrm{~nm}$, height of $\sim 2-3 \mathrm{~nm}$ for each individual nucleosome) can be consistently observed. Furthermore, superbeads (width of $\sim 40 \mathrm{~nm}$, height of $\sim 7 \mathrm{~nm}$ ) are demonstrated. Visualization of the solenoid conformation at the level of $30 \mathrm{~nm}$ chromatin fiber is attained either by using AFM or by using electron microscopy. In addition, tightly coiled chromatin fibers $(\sim 50-60 \mathrm{~nm}$ and $\sim 90$ $110 \mathrm{~nm}$ ) can be revealed. Our data suggest that the chromatin in the interphase nucleus of chicken erythrocyte represents a high-order conformation and AFM provides useful high-resolution structural information concerning the folding pattern of interphase chromatin fibers.
\end{abstract}

Key words: The chromatin folding pattern, chicken erythrocyte, atomic force microscopy.

\footnotetext{
1. To whom correspondence should be addressed: Shanghai Institute of Cell Biology, Chinese Academy of Sciences, 320 Yueyang Road, Shanghai 200031, China.
} 
The chromatin folding patterns in chicken erythrocytes by AFM

\section{INTRODUCTION}

Owing to the tremendous packing density and folding complexity in mitotic chromosomes, analysis of chromosome architecture has recently focused on interphase chromatin structure. To date, a number of models for the chromatin folding pattern have been proposed[1-6]. However, insufficient evidence has been obtained to confirm any of these model structures under native condition.

The atomic force microscope is now emerging as a useful tool in biological research, AFM can image biological molecules under conditions close to their native environment. So far it is the only-microscope that can achieve nanometer scale resolution on biological samples under native condition. Recently a number of different biological samples have been imaged including DNA and chromatin[7-11]. In some cases, AFM provides more clearly resolved images of the chromatin fibers than attained using electron microscopy.

It is our aim to use AFM to reveal the chromatin packing model within the nucleus of chicken erythrocytes. Evidence for the presence of the solenoid conformation at the level of $\sim 30 \mathrm{~nm}$ chromatin fiber is presented. However, the flat zig-zag ribbon was rarely observed. Furthermore, tightly coiled chromatin fibers $(\sim 50-60 \mathrm{~nm}$ and $\sim 90-110 \mathrm{~nm}$ ) could be demonstrated. Reproducible high-resolution AFM images of individual nucleosome and linker DNA could be revealed very clearly along chromatin fibers. In addition, surperbeads arranged with irregular distance along the extended beads-on-a string were visualized. Our data suggest that higher-order chromatin conformation might exist in the interphase chromatin.

\section{MATERIALS AND METHODS}

\section{Samples}

Preparation of samples for electron microscopy: Nuclei from chicken erythrocyte were isolated essentially as described by Zhu et al[12]. Nuclei were spread on water according to Gall, J.[13] with some modifications and fixed for $10 \mathrm{sec}$. with $2.5 \%$ glutaraldehyde, washed with PBS, then stained with $1 \%$ phospho-tungstic acid and 1\% urany! acetate in $70 \%$ EtOH, rinsed with water and air-dried.

The grids were metal-shadowed for electron microscopy analysis. In addition, sections of chromatin for TEM analysis were prepared and examined with a transmission electron microscope as described by Belmont et al.[14].

Atomic force microscopy of chromatin: For AFM analysis, isolated nuclei were spread on a nanopure water surface for 2, 5 and $10 \mathrm{~min}$. Chromatin was deposited on mica which was pretreated with 1\% APS (3-Aminopropyl Triethoxysilane). APS treatment markedly improved adhesion of spread chromatin to mica. Excess liquid was blown off with nitrogen gas. Tapping mode images were obtained on a nanoscope III AFM (Digital instruments, Santa Barbara, CA), using nanoprobe silicon tips, scan rate 1-1.5 HZ.

\section{RESULTS}

AFM images of the first level of DNA packing into nucleosome

Experiments were performed using the three-dimensional imaging capabilities of a 
Qian RL et al.

atomic force microscope, which makes the imaging of chromatin fibers possible under a native and less damaging conditions. After hypotonic lysis, the nuclear contents were released. Samples of native chromatin isolated from chicken erythrocytes were
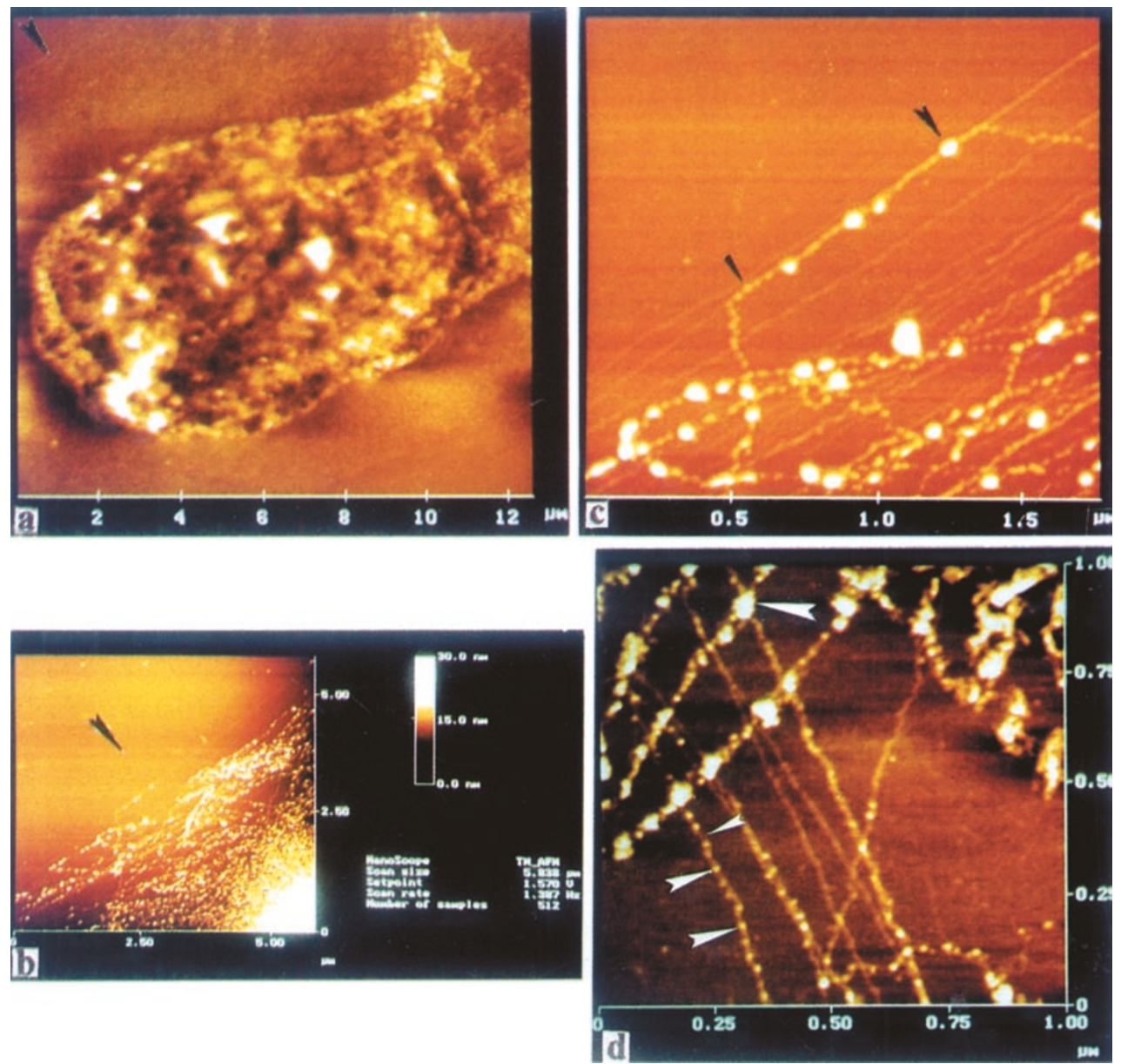

Fig 1. Chromatin from hypotonic lysed nucleus of chicken erythrocytes: (a) A membranous scaffold surrounded by dispersed chromatin. (b) Zoom into the outer region of chromatin layer indicated by arrow head in Fig 1a, in which single chromatin fibers appear. (c) Zoom into the regionas indicated with arrow head in Fig $1 \mathrm{~b}$. The beaded nucleosome structures are clearly visible. Small arrow head points to nucleosomes, while large one points to superbeads. (d) AFM image of spread chromatin in which single extented beads -on-a-string can be visualized very clearly, small arrow head points to linker DNA, middle ones point to nucleosomes and large one points to Superbeads. 
The chromatin folding patterns in chicken erythrocytes by AFM

spread onto freshly cleaved mica for AFM imaging. Nuclear membrane surrounded by dispersed chromatin were revealed in Fig 1a. More details of chromatin halo were demonstrated in Fig 1b-d. Single fibers were visible at the border of the halo. An extented beads -on a string structure could be repeatedly observed. Individual nucleosome cores and linker DNA could be resolved clearly along chromatin fibers (Fig 1b-d). The mean height of nucleosomes measured in tapping mode AFM was $\sim 2-3 \mathrm{~nm}$ and the mean width was $\sim 15-20 \mathrm{~nm}$. Our results are similar to those of W Fritzsche et al[15]. Furthermore, a superbeads conformation with irregular distance along chromatin fibers could be detected. The mean height of superbeads was $\sim 7 \mathrm{~nm}$ and the mean width was $\sim 40 \mathrm{~nm}$ (Fig 1c). We suggest that this kind of arrangement might be related to some biological function.

\section{AFM images of $\sim 30 \mathrm{~nm}$ chromatin fibers}

The $\sim 30 \mathrm{~nm}$ chromatin fibers observed in eukaryotic nuclei are considered as a discrete level in a hierarchy of DNA folding. However, the details of the higher order of folding in chromosomes and interphase nuclei are still incompletely understood. So far, a number of models have been proposed, including a solenoid model[16, 17], a helical ribbon model[18, 19] and others[20, 21]. However, insufficient evidence has been obtained to confirm any of these model structures under native condition. In the present study, a solenoid conformation has been revealed either by using AFM or by using electron microscopy. Especially, it can be clearly visualized in the more loosely folded and extended 30nm chromatin fibers (Fig 2, Fig 3). Fig 2 shows that the nucleosome core and linker DNA are tightly folded into $\sim 15 \mathrm{~nm}$ chromatin fiber. Then, $\sim 15 \mathrm{~nm}$ chromatin fiber is further folded into $\sim 30$ to $35 \mathrm{~nm}$ and $\sim$ $60 \mathrm{~nm}$ chromatin fibers.

The organization of the higher order chromatin fibers above the 30nm chromatin fibers in chicken erythrocytes

Belmont et al (1994) have used light microscopy and serial thin-section electron microscopy to examine the chromosome decondensation during G1 progression in synchronized CHO cells. Data showed that in early G1 nuclei 100-130nm chromonema fibers could be traced. However, more extented and less condensed 60-80 nm chromonema fibers could be predominantly detected in late G1 and early $\mathrm{S}$ phase nuclei[14]. We have used the same method to examine the nuclei of chicken erythrocyte, and the chromatin fibers in these nuclei were also visualized (data not shown).

In the present study, the organization of the higher order structure of interphase chromatin in chicken erythrocytes has been examined with AFM. After hypotonic lysis, nuclear contents were released. In some cases, largely intact chromatin was still retained (Fig 4). The clear images of the higher order structure of interphase chromatin in chicken erythrocyte were thus obtained. The chromatin fibers $(\sim 30$ - 
Qian RL et al.

Fig 2. Visualization of $30 \mathrm{~nm}$ chromatin fibers by using AFM. Chromatin fibers at three levels were revealed: Small arrow head marks the $\sim 15 \mathrm{~nm}$ chromatin fiber; middle one points to the folding of tightly packed, individual $30 \mathrm{~nm}$ chromatin fiber, chromatin is tightly organized into a cylinder conformation; large arrow head points to the $\sim 60 \mathrm{~nm}$ chromatin fiber.

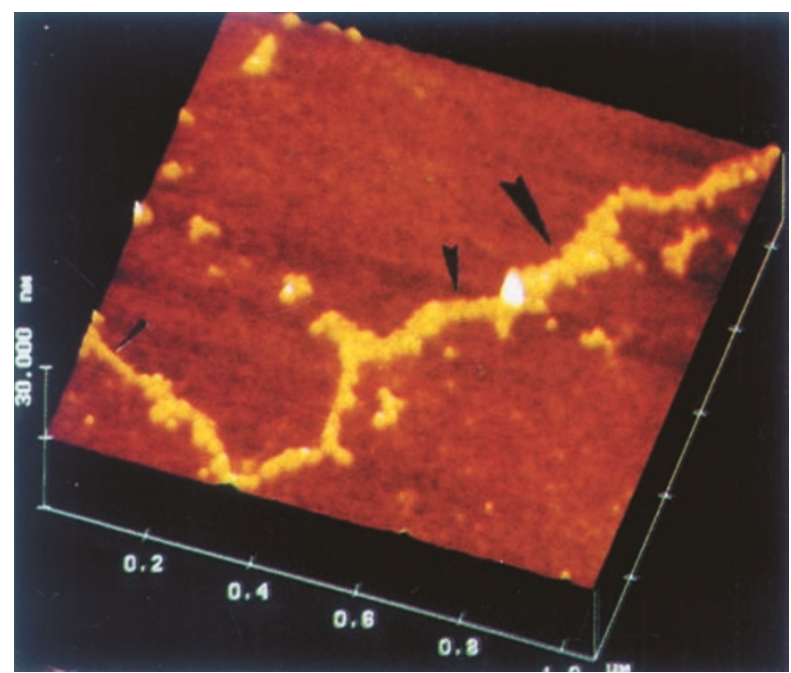

Fig 3. Identification of $30 \mathrm{~nm}$ chromatin fiber by using electron microscopy. (a) A small region of $30 \mathrm{~nm}$ chromatin fiber was demonstrated. Arrow head points to the folding pattern of $30 \mathrm{~nm}$ chromatin fiber with a solenoid model. $15,000 \times$ 8. (b) Arrow head points to the solenoid conformation. $15,000 \times 12$.

Fig 4. AFM images of chromatin from hypotonic lysed nuclei of chicken erythrocyte. After hypotonic lysis the contents released from the nucleus which still preserved a high condensation state. Some of the morphological features of erythrocyte chromatin were retained, enabling the imaging of chromatin structure.

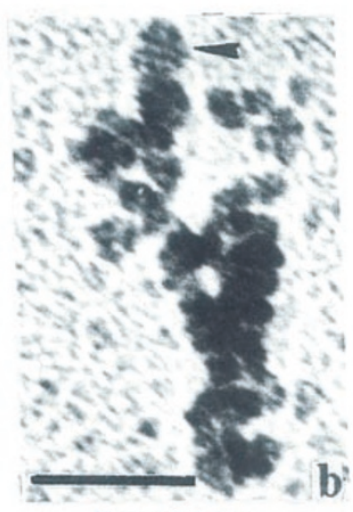

$\mathrm{Bar}=0.1 \mu \mathrm{m}$

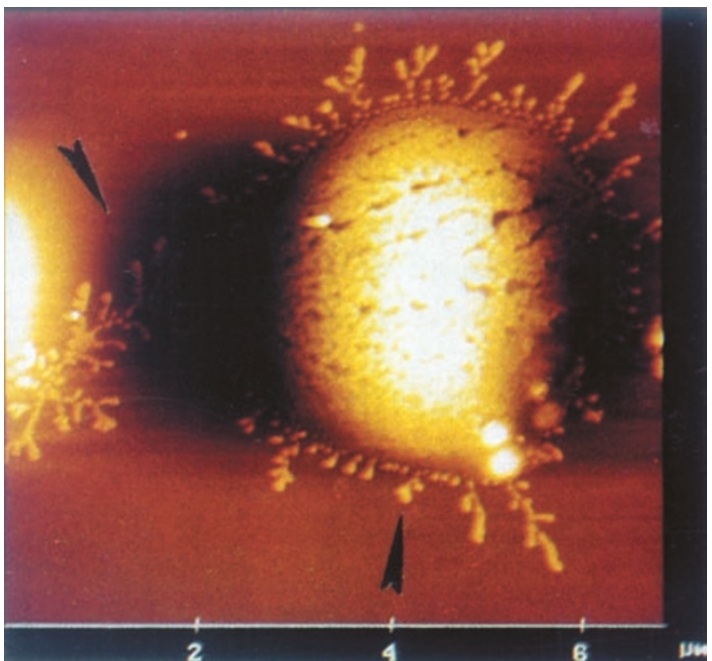


The chromatin folding patterns in chicken erythrocytes by AFM

$35 \mathrm{~nm}, \sim 50-60 \mathrm{~nm}$ and $\sim 90-110 \mathrm{~nm})$ as three higher-order interphase chromatin conformation could be visualized (Fig 5 a-d).

Furthermore, looping of chromonema fibers from the border region of the spherical chromatin could be visualized and chromatin fibers were folded in very symmetric model (data unpublished).

\section{DISCUSSION}

Owing to the limitation of techniques, the details of the higher order of chromatin folding, which predominate in interphase nuclei and chromosomes are still unclear. There is considerable debate as to how the nucleosomes and linker DNA are organized within chromatin fibers.

The goal of this study was to reveal the chromatin folding pattern in chicken erythrocyte nucleus by using a tapping mode scanning force microscopy, which can image native biological structure using simple, rapid sample preparations without the need for staining or metal coating. So far, it is the only microscope that can achieve nanometer-scale resolution on biological samples under native conditions. AFM is now emerging as a useful structural approach to study the higher order packaging of DNA in chromatin. In the present study, several levels of chromatin folding have been visualized in the nucleus of chicken erythrocytes. The "beads-on-a string" chromatin structure could be consistently observed. Interestingly, clear visualizations of the solenoid conformation $(\sim 30 \mathrm{~nm})$ were demonstrated either by using electron microscopy or by using AFM. In addition, owing to the milder spreading conditions, the higher order chromatin conformation could be revealed. Our data demonstrated that these higher order $30 \mathrm{~nm}$ chromatin fibers were further folded into $\sim 60 \mathrm{~nm}$ chromatin fibers. Then, $\sim 60 \mathrm{~nm}$ chromatin fibers were, in turn, folded into $\sim 90-110 \mathrm{~nm}$ and more larger chromatin fibers. We postulated that after hypotonic lysis the nuclear membrane was broken, and the nuclear contents were released from the chichen erythrocyte nuclei. In some cases the nuclear scaffold might be retained and the high order conformation of chromatin could be visualized.

From our data it appears that the interphase chromatin in the nucleus of chicken erythrocyte is not amorphous in texture, but can be folded into higher order chromatin conformation. In addition, a superbead structure could be occasionally visualized, especially in the loosely extended region of chromatin. We suggested that the conformation of superbeads might be related to a specific functional structural unit. It might be responsible for the gene expression and regulation.

\section{ACKNOWLEGEMENTS}

We are very grateful to Prof. WANG Ya-Hui for helpful suggestions and for critical reading of the manuscript. We also thank GAO Qi-Rong for excellent assistance in preparing the electron microscopic pictures. This project was supported by grants from National Natural Science Foundation, and from Chinese Academy of Sciences. 
Qian RL et al.
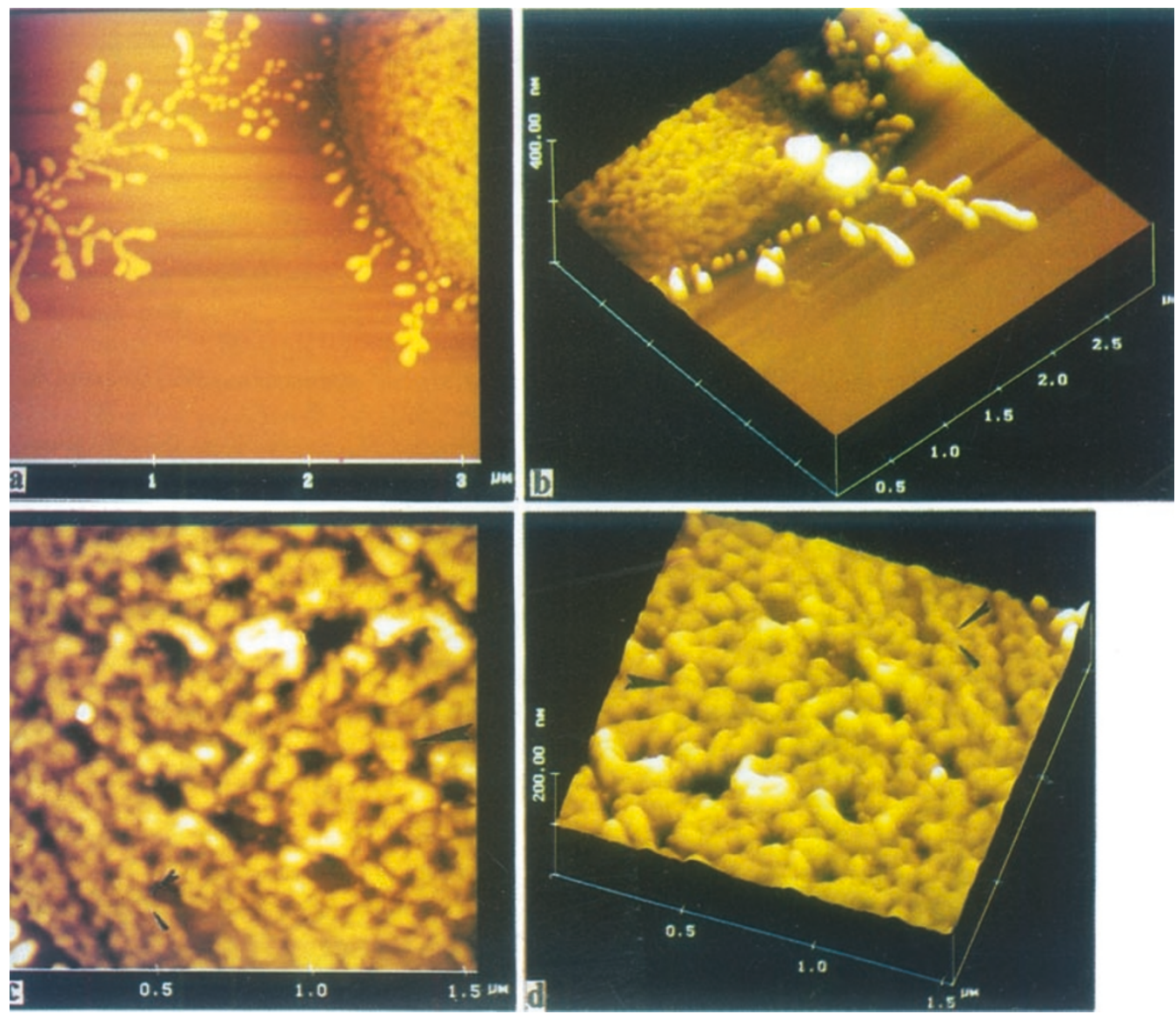

Fig 5. Visualization of levels of chromatin folding above the $30 \mathrm{~nm}$ chromatin fiber in chicken erythrocyte by using AFM. (a) and (b), Zoom of a border region of the spherical contents indicated with arrow heads in Fig 4. (c) and (d), Zoom of a middle region of the spherical contents in Fig 4, $\sim 30-35 \mathrm{~nm}$ (small arrow heads), $\sim 50-60 \mathrm{~nm}$ (middle arrow heads) and $\sim 90-110 \mathrm{~nm}$ (large arrow heads) chromatin fibers were revealed.

\section{REFERENCES}

[1] Sedat J, L Manuelidis. A direct approach to the structure of eukaryotic chromosomes. Cold spring harbor symp. Quant Biol 1997; 42:331-50.

[2] Paulson JR, UK Laemmli. The structure of histone depleted metaphase chromosomes. Cell $1977 ; \mathbf{1 2}: 817-28$. 
[3] Marsden MPF, UK Laemmli. Metaphase chromosome structure: evidence for a radial loop model. Cell 1979; 17:849-58.

[4] Adolph KW. Organization of chromosomes in mitotic HeLa cells. Exp Cell Res 1980; 125:95103.

[5] Rattner JB, CC Lin. Radial loops and helical coils coexist in metaphase chromosomes. Cell 1985; 42:291-6.

[6] Boy de la Tour E, UK Laemmli. The metaphase scaffold is helically folded: sister chromatids have predominantly opposite helical handedness. Cell 1988; 55:937-44.

[7] Leuba SH, Guoliang Yang, Charles Robert, Bruno Samori, Kensal van Holde, Jordanka Zlatanova, Carlos Bustamante. Three-dimensional structure of extended chromatin fibers as revealed by tapping-mode scanning force microscopy. Proc Natl Acad Sci USA 1994; 91:11621-5.

[8] Zlatanova J, Leuba SH, Guoliang Yang, Carlos Bustamante, Kensal van Holde. Linker DNA accessibility in chromatin fibers of different conformations: A reevaluation. Proc Natl Acad Sci USA 1994; 91:5277-80.

[9] Martin LD, Vesenka JP, Henderson E, Dobbs DL. Visualization of nucleosomal substructure in native chromatin by atomic force microscopy. Biochemistry 1995; 34:4610-6.

[10] Guoliang Yang, Sanford H Leuba, Carlos Bustamante, Jordanka Zlatanova, Kensal Van Holde. Role of linker histones in extended chromatin fibre structure. Structural Biology 1994; 1:761-3.

[11] Lyubchenko YL. et al Atomic force microscopy of DNA and bacteriophage in air, water and propanol: the role of adhesion forces. Nucleic Acids Res 1993; 21:1117-23.

[12] Jing-de Zhu, Xiao-ping Sun, Fan Wang. The DNA intercalator, ethidium bromide, alters the pattern of DNase I hypersensitive sites of the $\beta^{A}$-globin gene in chicken erythrocytes. Biochem Biophys Acta 1991; 1089:158-66.

[13] Gall J. Chromosome fibers from an interphase nucleus. Science 1963; 139:120-1.

[14] Belmont AS, Bruce K. Visualization of G1 chromosomes: A folded, Twisted, Supercoiled chromonema model of interphase chromatid structure. J Cell Biol 1994; 127:287-302.

[15] Fritzsche W, Schaper A, Jovin TM. Probing chromatin with the scanning force microscope. Chromosoma 1994; 103:231-6.

[16] Finch JT, Klug A. Solenoidal model for superstructure in chromatin. Proc Natl Acad Sci USA 1976; 73:1897-901.

[17] McGhee JD, Rau DC, Charney E, Felsenfeld G. Orientation of the nucleosome within the higher order structure of chromatin. Cell 1980; 22:87-96.

[18] Worcel A, Strogatz S, Riley D. Structure of chromatin and the linking number of DNA. Proc Natl Acad Sci USA 1981; 78:1461-5.

[19] Woodcock CLF, Frado L-LY, Rattner JB. The higher-order structure of chromatin: Evidence for a helical ribbon arrangement. J Cell Biol 1984; 99:42-52.

[20] Woodcock CL, Grigoryev SA, Horowitz RA, Whitaker N. A chromatin folding model that incorporates linker variability generates fibers resembling the native structures. Proc Natl Acad Sci USA 1993; 90:9021-5.

[21] Horowitz RA, Agard DA, Sedat JW, Woodcock CL. The three-dimensional architecture of chromatin in situ: Electron tomography reveals fibers composed of a continuously variable Zig-Zag nucleosomal ribbon. J Cell Biol 1994; 125:1-10.

Received 22-8-1997. Revised 21-9-1997. Accepted 24-9-1997. 